\title{
Fluctuation of fasting blood glucose in patients who underwent primary or revision total joint arthroplasty: a retrospective review
}

Yongyu Ye, Baiqi Pan, Minghui Gu, Guoyan Xian, Weishen Chen, Linli Zheng, Ziji Zhang* and Puyi Sheng*(D)

\begin{abstract}
Background: Perioperative hyperglycemia is a risk factor for postoperative complications after total joint arthroplasty (TJA). However, the variability of fasting blood glucose (FBG) after TJA remains unknown. We aimed to assess the fluctuation and extent of elevation of FBG following primary or revision TJA.

Methods: We retrospectively evaluated the medical records of 1788 patients who underwent primary or revision TJA between 2013 and 2018. We examined FBG values collected during 6 days of the perioperative period. The findings for each time point were evaluated with descriptive statistics. Postoperative glycemic variability was assessed by the coefficient of variation (CV).

Results: The final cohort included the medical records of 1480 patients (1417 primary and 63 revision). FBG was highest on postoperative day 1 in the primary and revision groups $(P<0.001)$, which had the highest number of hyperglycemic patients (FBG > $100 \mathrm{mg} / \mathrm{dL}$ ), with $66.4 \%$ and $75.5 \%$ in the primary and revision groups, respectively. The CV of diabetics in the primary group, and diabetics and non-diabetics in the revision group, was higher than that of non-diabetics in the primary group.

Conclusion: Postoperative day 1 showed the highest FBG levels and proportion of patients with hyperglycemia in the perioperative period. Primary group diabetics, and revision group diabetics and non-diabetics, had higher postoperative fluctuation of FBG than primary group non-diabetics. Frequent FBG monitoring may therefore be warranted in diabetic patients undergoing TJA, and all patients undergoing revision TJA.
\end{abstract}

Keywords: Total joint arthroplasty, Revision, Fasting blood glucose, Fluctuation, Hyperglycemia

\section{Background}

Total joint arthroplasty (TJA) is a surgical procedure to treat late-stage osteoarthritis and improve the quality of life [1]. However, more complications are being recorded than previously, due to the increased number of TJA surgeries $[2,3]$. Risk factors for postoperative complications,

\footnotetext{
* Correspondence: zhangziji@mail.sysu.edu.cn; shengpy@mail.sysu.edu.cn; shengpuyi@hotmail.com

Department of Orthopedic Surgery, The First Affiliated Hospital, Sun Yat-sen University, 58 Zhongshan 2nd Road, Guangzhou 510080, China
}

including age, obesity, malnutrition, prior infection, and hyperglycemia, were demonstrated by several studies [46]. Nonetheless, translation of these factors into clinical practice to avoid complications and reach promising outcomes has been addressed critically important [7-10]. For example, correction of malnutrition and controlling the blood glucose within normal levels would have a great impact on minimizing postoperative complications $[11,12]$.

Recent studies have confirmed hyperglycemia as a significant risk factor for postoperative complications in

(c) The Author(s). 2020 Open Access This article is licensed under a Creative Commons Attribution 4.0 International License, which permits use, sharing, adaptation, distribution and reproduction in any medium or format, as long as you give appropriate credit to the original author(s) and the source, provide a link to the Creative Commons licence, and indicate if changes were made. The images or other third party material in this article are included in the article's Creative Commons licence, unless indicated otherwise in a credit line to the material. If material is not included in the article's Creative Commons licence and your intended use is not permitted by statutory regulation or exceeds the permitted use, you will need to obtain permission directly from the copyright holder. To view a copy of this licence, visit http://creativecommons.org/licenses/by/4.0/. The Creative Commons Public Domain Dedication waiver (http://creativecommons.org/publicdomain/zero/1.0/) applies to the data made available in this article, unless otherwise stated in a credit line to the data. 
TJA, independent of diabetes mellitus (DM) status $[4,6$, 13]. Timely recognition of fluctuation of glucose levels and maintaining glycemic control is crucial for reducing undesired complications [6]. Kheir et al. noted a linear relationship between postoperative blood glucose and periprosthetic joint infection (PJI) [4], while Shohat et al. demonstrated that higher postoperative glucose variability was associated with increased rates of complications [6]. Varady et al. suggested that $9 \mathrm{pm}$ on the night of TJA surgery was the most sensitive time within $24 \mathrm{~h}$ for detecting hyperglycemia in both diabetic and nondiabetic patients [14]. Though some researchers have demonstrated the variability of glucose levels in patients undergoing TJA and acute fluctuation of blood glucose within $24 \mathrm{~h}$ following TJA, an exact conclusion regarding fasting blood glucose (FBG) fluctuation and the extent to which FBG may become elevated postoperatively in TJA patients remains unknown.

Therefore, we investigated the variability and distribution of FBG during 6 days postoperatively in patients undergoing primary or revision TJA to explore the differences and fluctuation of FBG, which may aid clinicians in controlling FBG in various aspects of clinical practice.

\section{Materials and methods}

\section{Selection criteria and data review}

Following approval by our institutional review board, this retrospective study reviewed 1788 medical records of patients who were admitted to the orthopedic department of our hospital between October 2013 and November 2018. We included patients who underwent total knee arthroplasty (TKA) or total hip arthroplasty (THA) as either a primary or revision surgical procedure. The exclusion criteria were as follows: (1) patients with underlying inflammatory conditions, e.g., rheumatoid arthritis and ankylosing spondylitis, as this would impact blood glucose levels; (2) patients with malignancy, as evidence showed patients with malignancy had higher blood glucose levels [15]; (3) no previous surgical record; (4) lack of FBG data within 6 days postoperatively. Finally, the medical records of 1417 primary and 63 revision TJA patients were selected for the study. Demographic information (age, sex, type of procedure, date of admission, date of discharge), comorbidities (malignancy, rheumatoid arthritis, ankylosing spondylitis, DM, and hypertension), operative details (type of anesthesia, date of surgery, start time, end time, and duration of surgery), and reasons for surgery (PJI, periprosthetic osteolysis, aseptic loosening, periprosthetic fracture, dislocation, and surgical site infection [SSI]) were extracted from the records. The definitions of SSI and PJI were based on the Centers for Disease Control (CDC) definition and the Musculoskeletal Infection
Society criteria [16, 17]. The medial parapatellar approach was used for TKA, and the posterior lateral approach was used for THA. The requirement for informed consent was waived due to the retrospective design of our study.

\section{Laboratory evaluation and management protocol}

Routine blood tests, including basic metabolic panel, were ordered preoperatively for all patients admitted for arthroplasty. FBG values were based on serum glucose levels in the basic metabolic panel preoperatively and during the 6 days postoperatively. All samples were drawn at approximately 7 am after patients had fasted overnight for a minimum of $8 \mathrm{~h}$. The diagnosis of DM was based on past medical history or the American Diabetes Association (ADA) criteria regarding the blood glucose after admission. Patients with DM were given diabetic meals, and those without were given standard meals, from the hospital canteen. All patients were on restricted solids and liquids for at least $8 \mathrm{~h}$ before surgery. Either general or spinal anesthesia was performed. Postoperatively, most patients received an analgesic pump. All patients were given lactated Ringer's solution or glucose saline intravenously within $6 \mathrm{~h}$ following surgery. In case of hyperglycemia resulting from the infusion of glucose, supplementary insulin was added to the glucose saline for diabetic patients. Patients with DM were initiated on sliding-scale insulin therapy or antidiabetic drugs. Fixed-amount carbohydrate meals were provided to patients with DM at each meal from the hospital canteen. Patients with DM underwent regular fingertip glucose level monitoring and adjustment of oral medications or insulin according to their glucose levels. Non-steroidal antiinflammatory drugs such as loxoprofen or celecoxib were used to relieve pain postoperatively. Routine blood tests postoperative were performed for all patients. Ordering of blood tests on other days was determined by the doctors in charge and the general status of patients. After discharge, patients underwent regular follow-up.

\section{Classification of FBG}

We classified FBG levels into several categories to identify the hyperglycemic state of each patient at each time point, based on previous studies [4, 13, 14, 18-22]. According to the definition from the ADA and evidence published by Varady et al. [14, 18], the normal status was defined as FBG < $100 \mathrm{mg} / \mathrm{dL}$. FBG between $100 \mathrm{mg} /$ $\mathrm{dL}$ and $126 \mathrm{mg} / \mathrm{dL}$ was defined as elevated blood glucose. The other three categories for FBG used to define hyperglycemia were as follows: strict $>126 \mathrm{mg} / \mathrm{dL}$, intermediate $>137 \mathrm{mg} / \mathrm{dL}$, and lenient $>180 \mathrm{mg} / \mathrm{dL}$. Postoperative glycemic variability was evaluated by calculating the coefficient of variation $(\mathrm{CV})$, which is the ratio of the standard deviation to the mean FBG [23, 24]. 


\section{Statistical analysis}

For the demographic features, age differences were analyzed using the independent $t$ test, and categorical variables were calculated using the chi-square test. The Mann-Whitney $U$ test was applied for divergence in glycemic status. The measures of FBG (mean, standard deviation, median, range, and hyperglycemic rate) were calculated at each time point. The variability and fluctuation of FBG were revealed by boxplot and CV. To evaluate the differences between each pair of time points, we used the Kruskal-Wallis $H$ test for comparisons, and the $P$ value was adjusted by Bonferroni analysis. A $P$ value $<0.05$ was considered statistically significant. All analyses were performed using SPSS (Statistical Package for the Social Sciences) version 22 (IBM Corporation, Armonk, New York).

\section{Results}

\section{Demographic features and general glycemic status}

Medical records of 1480 patients (1417 primary and 63 revision) were studied. There were no significant differences in age and sex ratio between the two groups $(P=$ 0.17 and $P=0.10$, respectively). In the revision group, the number of patients with hip problems was significantly higher than that in the primary group $(P<0.001)$. For DM, hypertension, and anesthesia, no significant differences were revealed between the primary and revision groups. For the glycemic status of FBG, significant differences were noted between primary and revision patients postoperatively $(P=0.01)$ (Table 1$)$.

\section{The fluctuation of perioperative FBG}

\section{Primary total joint arthroplasty patients}

Among all patients (diabetic and non-diabetic together) or non-diabetic group with primary TJA, the mean glucose levels at POD1 (postoperative day 1) were significantly higher than that at all other time points $(P<$ $0.001)$. There were no significant differences in FBG between PODs 2 and 3, PODs 3 and 4, PODs 4 and 5, or PODs 5 and 6. From PODs 1 to 6, FBG gradually declined and became stable (CVs of all patients and nondiabetic patients: $7.14 \%$ and $6.85 \%$ respectively). Further examination of the primary group revealed that FBG at POD1 in diabetics was only significantly higher than that measured preoperatively $(P<0.001)$, and the variability of FBG was higher in diabetics within the primary group (CV\%, 9.02) (Table 2, Fig. 1).

We further classified hyperglycemia into four groups. POD1 had the highest number of hyperglycemic patients (66.4\% for FBG > $100 \mathrm{mg} / \mathrm{dL}, 37.0 \%$ for FBG of $100-126$ $\mathrm{mg} / \mathrm{dL}$, and $29.4 \%$ for $\mathrm{FBG}>126 \mathrm{mg} / \mathrm{dL}$ ). When grouped by diabetic status, $63.7 \%$ of non-diabetic and $85.0 \%$ of diabetic patients were hyperglycemic (> 100 $\mathrm{mg} / \mathrm{dL}$ ) at POD1 (Table 2).
Table 1 Demographic features and glycemic status of patients who underwent total joint arthroplasty

\begin{tabular}{|c|c|c|c|}
\hline Group and variable & Primary & Revision & $P$ value \\
\hline No. of patients & 1417 & 63 & \\
\hline $\mathrm{Age}^{\mathrm{a}}$ & $63.3 \pm 12.3$ & $65.5 \pm 11.1$ & $0.17^{c}$ \\
\hline $\operatorname{Sex}^{\mathrm{b}}$ & & & $0.10^{\mathrm{d}}$ \\
\hline Female & $1012(71.4 \%)$ & $39(61.9 \%)$ & \\
\hline Male & $405(28.6 \%)$ & $24(38.1 \%)$ & \\
\hline Joint ${ }^{b}$ & & & $<0.001^{d}$ \\
\hline Hip & $604(42.6 \%)$ & $53(84.1 \%)$ & \\
\hline Knee & $813(57.4 \%)$ & $10(15.9 \%)$ & \\
\hline Diabetes mellitus ${ }^{b}$ & & & $0.17^{d}$ \\
\hline Diabetic & $184(13.0 \%)$ & $12(19.0 \%)$ & \\
\hline Non-diabetic & $1233(87.0 \%)$ & $51(81.0 \%)$ & \\
\hline Blood pressure ${ }^{b}$ & & & $0.23^{d}$ \\
\hline Hypertension & $580(40.9 \%)$ & $42(66.7 \%)$ & \\
\hline Non-hypertension & $837(59.1 \%)$ & $21(33.3 \%)$ & \\
\hline Anaesthesia $^{\mathrm{b}}$ & & & $0.28^{d}$ \\
\hline Spinal & $1032(72.8 \%)$ & $43(68.3 \%)$ & \\
\hline General & $385(27.2 \%)$ & $20(31.7 \%)$ & \\
\hline \multicolumn{4}{|l|}{ Glycemic status (mg/dL) ${ }^{a}$} \\
\hline Pre-operation & $93 \pm 26$ & $94 \pm 28$ & $0.81^{e}$ \\
\hline Post-operation (within 6 days) & $109 \pm 32$ & $121 \pm 45$ & $0.01^{\mathrm{e}}$ \\
\hline Total & $103 \pm 31$ & $111 \pm 42$ & $0.55^{\mathrm{e}}$ \\
\hline
\end{tabular}

TJA total joint arthroplasty

${ }^{a}$ Data are presented as the mean \pm standard deviation

${ }^{b}$ Data are presented as the number (percentage) of patients

${ }^{c} P$ value was calculated by the independent $t$ test

${ }^{d} P$ value was calculated by the chi-square test

${ }^{\mathrm{e}} P$ value was calculated by the Mann-Whitney $U$ test. $P<0.05$ indicates a significant difference between groups

\section{Revision surgery patients}

In the revision group, FBG at POD1 in all patients (diabetic and non-diabetic together) or non-diabetic group was significantly higher than at the PRDs (preoperative days) $(P<0.001)$. There were no significant differences in FBG between PODs 2 and 3, PODs 3 and 4, PODs 4 and 5 , or PODs 5 and 6 . The CVs of all patients and non-diabetic patients were $8.82 \%$ and $12.83 \%$ postoperatively, and FBG displayed large fluctuation. However, compared to the non-diabetic group, there were no significant differences in FBG between each time point in the diabetic group, and the variability of FBG was higher (CV\%, 15.31) (Table 3, Fig. 2).

The highest number of hyperglycemic $(>100 \mathrm{mg} / \mathrm{dL})$ patients in the revision group was at POD1, which was far higher than that on the other days. When stratified by diabetic status, $69.1 \%$ of non-diabetic and $100 \%$ of diabetic patients were hyperglycemic (> $100 \mathrm{mg} / \mathrm{dL}$ ) at POD1 (Table 3). 
Table 2 Perioperative glucose levels of the primary total joint arthroplasty population

\begin{tabular}{|c|c|c|c|c|c|c|c|c|c|c|}
\hline \multirow[t]{2}{*}{ Day } & \multirow{2}{*}{$\begin{array}{l}\text { Cases } \\
(n)\end{array}$} & \multicolumn{8}{|c|}{ Glucose level (mg/dL) } & \multirow{2}{*}{$\begin{array}{l}\mathrm{CV} \\
(\%)^{\mathrm{b}}\end{array}$} \\
\hline & & $\begin{array}{l}\text { Mean } \\
\pm \text { SD }\end{array}$ & Median & Range & $\begin{array}{l}\text { Normal }^{\mathrm{a}}(< \\
100 \mathrm{mg} / \mathrm{dL})\end{array}$ & $\begin{array}{l}\text { Elevated }^{\mathrm{a}}(100- \\
126 \mathrm{mg} / \mathrm{dL})\end{array}$ & $\begin{array}{l}\text { Strict }^{\mathrm{a}}(>126 \\
\mathrm{mg} / \mathrm{dL})\end{array}$ & $\begin{array}{l}\text { Intermediate }{ }^{\mathrm{a}} \text { (> } \\
137 \mathrm{mg} / \mathrm{dL})\end{array}$ & $\begin{array}{l}\text { Lenient }^{\mathrm{a}}(> \\
180 \mathrm{mg} / \mathrm{dL})\end{array}$ & \\
\hline \multicolumn{11}{|l|}{ Total } \\
\hline PRD & 1463 & $93 \pm 26$ & 88 & $47-367$ & $1133(77.4 \%)$ & $229(15.7 \%)$ & $101(6.9 \%)$ & $69(4.7 \%)$ & $20(1.4 \%)$ & \\
\hline POD1 & 1158 & $\begin{array}{l}117 \pm \\
35\end{array}$ & 110 & $40-383$ & $388(33.5 \%)$ & $429(37.0 \%)$ & $341(29.4 \%)$ & $228(19.7 \%)$ & $58(5.0 \%)$ & 7.14 \\
\hline POD2 & 470 & $\begin{array}{l}104 \pm \\
26\end{array}$ & 97 & $45-227$ & $255(54.3 \%)$ & $154(32.8 \%)$ & $61(13.0 \%)$ & $45(9.6 \%)$ & $10(2.1 \%)$ & \\
\hline POD3 & 217 & $\begin{array}{l}105 \pm \\
29\end{array}$ & 97 & $67-239$ & $119(54.8 \%)$ & $66(30.4 \%)$ & $32(14.7 \%)$ & $22(10.1 \%)$ & $9(4.1 \%)$ & \\
\hline POD4 & 205 & $\begin{array}{l}100 \pm \\
24\end{array}$ & 94 & $54-202$ & $132(64.4 \%)$ & $46(22.4 \%)$ & $27(13.2 \%)$ & $18(8.8 \%)$ & $4(2.0 \%)$ & \\
\hline POD5 & 177 & $96 \pm 21$ & 94 & $61-238$ & $126(71.2 \%)$ & $40(22.6 \%)$ & $11(6.2 \%)$ & $7(4.0 \%)$ & $2(1.1 \%)$ & \\
\hline POD6 & 143 & $99 \pm 38$ & 92 & $52-391$ & 99 (69.2\%) & $26(18.2 \%)$ & $18(12.6 \%)$ & $13(9.1 \%)$ & $4(2.8 \%)$ & \\
\hline \multicolumn{11}{|l|}{ Diabetic } \\
\hline PRD & 194 & $\begin{array}{l}118 \pm \\
45\end{array}$ & 109 & $47-317$ & $81(41.8 \%)$ & $53(27.3 \%)$ & $60(30.9 \%)$ & $45(23.2 \%)$ & $14(7.2 \%)$ & \\
\hline POD1 & 153 & $\begin{array}{l}144 \pm \\
47\end{array}$ & 139 & $40-364$ & $23(15.0 \%)$ & 35 (22.9\%) & $95(62.1 \%)$ & 77 (50.3\%) & $26(17.0 \%)$ & 9.02 \\
\hline POD2 & 59 & $\begin{array}{l}132 \pm \\
40\end{array}$ & 131 & $58-227$ & 15 (25.4\%) & $14(23.7 \%)$ & $30(50.8 \%)$ & $26(44.1 \%)$ & $7(11.9 \%)$ & \\
\hline POD3 & 29 & $\begin{array}{l}127 \pm \\
41\end{array}$ & 115 & $79-230$ & 10 (34.5\%) & $6(20.7 \%)$ & $13(44.8 \%)$ & $8(27.6 \%)$ & $5(17.2 \%)$ & \\
\hline POD4 & 36 & $\begin{array}{l}116 \pm \\
35\end{array}$ & 114 & $54-202$ & $13(36.1 \%)$ & $8(22.2 \%)$ & 15 (41.7\%) & $9(25.0 \%)$ & $3(8.3 \%)$ & \\
\hline POD5 & 19 & $\begin{array}{l}113 \pm \\
26\end{array}$ & 103 & $83-182$ & $6(31.6 \%)$ & $9(47.4 \%)$ & $4(21.1 \%)$ & $3(15.8 \%)$ & $1(5.3 \%)$ & \\
\hline POD6 & 17 & $\begin{array}{l}125 \pm \\
77\end{array}$ & 101 & $52-391$ & $8(47.1 \%)$ & $4(23.5 \%)$ & $5(29.4 \%)$ & $5(29.4 \%)$ & $2(11.8 \%)$ & \\
\hline \multicolumn{11}{|c|}{ Non-diabetic } \\
\hline PRD & 1269 & $90 \pm 18$ & 86 & $52-367$ & 1052 (82.9\%) & $176(13.9 \%)$ & $41(3.2 \%)$ & $24(1.9 \%)$ & $6(0.5 \%)$ & \\
\hline POD1 & 1005 & $\begin{array}{l}113 \pm \\
30\end{array}$ & 108 & $58-383$ & $365(36.3 \%)$ & 394 (39.2\%) & $246(24.5 \%)$ & $151(15.0 \%)$ & $32(3.2 \%)$ & 6.85 \\
\hline POD2 & 411 & $\begin{array}{l}100 \pm \\
21\end{array}$ & 97 & $45-205$ & $240(58.4 \%)$ & $140(34.1 \%)$ & $31(7.5 \%)$ & $19(4.6 \%)$ & $3(0.7 \%)$ & \\
\hline POD3 & 188 & $\begin{array}{l}101 \pm \\
24\end{array}$ & 95 & $67-239$ & 109 (58.0\%) & 60 (31.9\%) & 19 (10.1\%) & $14(7.4 \%)$ & $4(2.1 \%)$ & \\
\hline POD4 & 169 & $97 \pm 20$ & 94 & $65-191$ & $119(70.4 \%)$ & $38(22.5 \%)$ & $12(7.1 \%)$ & $9(5.3 \%)$ & $1(0.6 \%)$ & \\
\hline POD5 & 158 & $94 \pm 20$ & 90 & $61-238$ & $120(75.9 \%)$ & 31 (19.6\%) & 7 (4.4\%) & $4(2.5 \%)$ & $1(0.6 \%)$ & \\
\hline POD6 & 126 & $96 \pm 28$ & 90 & $63-306$ & 91 (72.2\%) & $22(17.5 \%)$ & $13(10.3 \%)$ & $8(6.3 \%)$ & $2(1.6 \%)$ & \\
\hline
\end{tabular}

$P R D$ pre-operative day, $P O D$ post-operative day, $S D$ standard deviation

${ }^{a}$ Data are presented as the number (percentage) of measurement

${ }^{b}$ Post-operative glycemic variability was assessed using a coefficient of variation (the ratio of the standard deviation to the mean glucose level)

\section{Discussion}

The clear association between perioperative hyperglycemia and postoperative complications (for example, PJI) after TJA has been established by previous studies
[4, 6, 25-27]. However, the fluctuation and extent of elevation of FBG within 1 week after TJA remains uncertain. In our study, we studied the FBG monitoring records through 6 days following TJA. We found that 


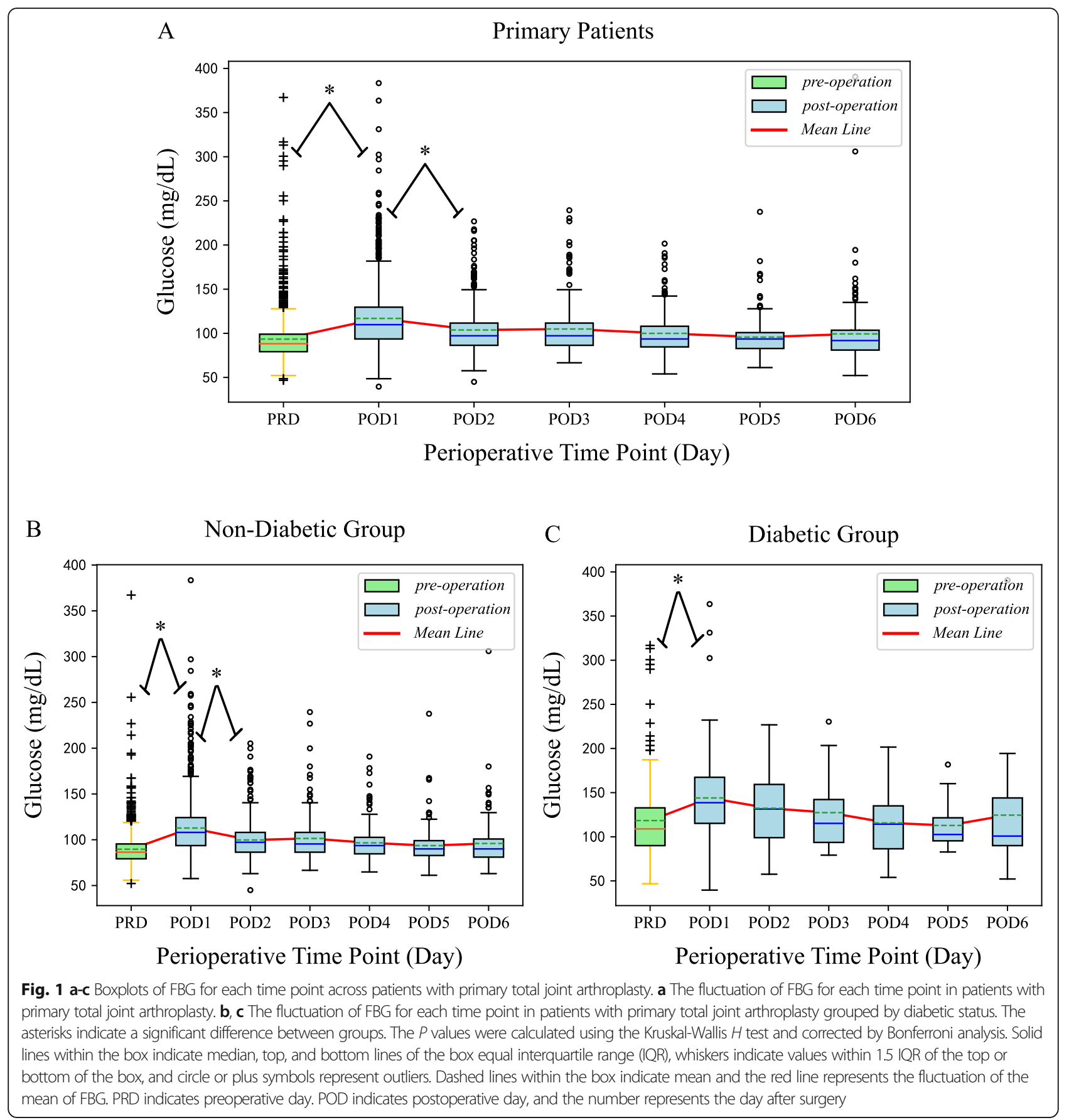

POD1 had the highest FBG level and the most hyperglycemic patients perioperatively, suggesting that investigating FBG at this time point may be warranted. Additionally, compared to non-diabetic patients in the primary group, the fluctuation of FBG was greater in patients with DM within the primary group and diabetic and non-diabetic patients in the revision group, indicating that frequent perioperative FBG assessment was useful for those patients.

Hyperglycemia, which can even occur in non-diabetics, was considered to be a risk factor for postoperative complications $[4,14,20]$. Notably, evidence shows that monitoring blood glucose levels and assessing glucose variability are effective in predicting complications [6]. Two studies showed that early postoperative glucose control could be a reducing factor in nosocomial infections [28, 29]. Specifically, Varady et al. examined the blood glucose values within POD1 in patients who underwent TJA and found that $9 \mathrm{pm}$ on the night of surgery showed the highest number of hyperglycemic patients, suggesting that this time point may be the most sensitive for detecting 
Table 3 Perioperative glucose levels of patients with revision surgery

\begin{tabular}{|c|c|c|c|c|c|c|c|c|c|c|}
\hline \multirow[t]{2}{*}{ Day } & \multirow{2}{*}{$\begin{array}{l}\text { Cases } \\
(n)\end{array}$} & \multicolumn{8}{|c|}{ Glucose level (mg/dL) } & \multirow{2}{*}{$\begin{array}{l}\text { CV } \\
(\%)^{b}\end{array}$} \\
\hline & & $\begin{array}{l}\text { Mean } \\
\pm \text { SD }\end{array}$ & Median & Range & $\begin{array}{l}\text { Normal }^{\mathrm{a}}(< \\
100 \mathrm{mg} / \mathrm{dL})\end{array}$ & $\begin{array}{l}\text { Elevated }^{\mathrm{a}}(100- \\
126 \mathrm{mg} / \mathrm{dL})\end{array}$ & $\begin{array}{l}\text { Strict }^{\mathrm{a}}(>126 \\
\mathrm{mg} / \mathrm{dL} \text { ) }\end{array}$ & $\begin{array}{l}\text { Intermediate }{ }^{\mathrm{a}} \text { (> } \\
137 \mathrm{mg} / \mathrm{dL})\end{array}$ & $\begin{array}{l}\text { Lenient }^{\mathrm{a}}(> \\
180 \mathrm{mg} / \mathrm{dL})\end{array}$ & \\
\hline \multicolumn{11}{|l|}{ Total } \\
\hline PRD & 66 & $94 \pm 28$ & 86 & $65-265$ & $50(75.8 \%)$ & $11(16.7 \%)$ & $5(7.6 \%)$ & $3(4.5 \%)$ & $1(1.5 \%)$ & \\
\hline POD1 & 53 & $\begin{array}{l}132 \pm \\
50\end{array}$ & 121 & $76-337$ & $13(24.5 \%)$ & $14(26.4 \%)$ & $26(49.1 \%)$ & $16(30.2 \%)$ & $6(11.3 \%)$ & 8.82 \\
\hline POD2 & 17 & $\begin{array}{l}110 \pm \\
39\end{array}$ & 101 & $68-194$ & $7(41.2 \%)$ & $6(35.3 \%)$ & $4(23.5 \%)$ & $4(23.5 \%)$ & $1(5.9 \%)$ & \\
\hline POD3 & 16 & $\begin{array}{l}107 \pm \\
26\end{array}$ & 102 & $77-184$ & $8(50.0 \%)$ & $6(37.5 \%)$ & $2(12.5 \%)$ & $1(6.2 \%)$ & $1(6.2 \%)$ & \\
\hline POD4 & 15 & $\begin{array}{l}108 \pm \\
33\end{array}$ & 92 & $74-180$ & $9(60.0 \%)$ & $3(20.0 \%)$ & $3(20.0 \%)$ & $3(20.0 \%)$ & $1(6.7 \%)$ & \\
\hline POD5 & 11 & $\begin{array}{l}116 \pm \\
56\end{array}$ & 97 & $65-230$ & $6(54.5 \%)$ & $3(27.3 \%)$ & $2(18.2 \%)$ & $2(18.2 \%)$ & $2(18.2 \%)$ & \\
\hline POD6 & 8 & $\begin{array}{l}126 \pm \\
46\end{array}$ & 109 & $77-189$ & $4(50.0 \%)$ & $1(12.5 \%)$ & $3(37.5 \%)$ & $3(37.5 \%)$ & $2(25.0 \%)$ & \\
\hline \multicolumn{11}{|l|}{ Diabetic } \\
\hline PRD & 14 & $\begin{array}{l}117 \pm \\
51\end{array}$ & 104 & $70-265$ & $6(42.9 \%)$ & $3(21.4 \%)$ & $5(35.7 \%)$ & $3(21.4 \%)$ & $1(7.1 \%)$ & \\
\hline POD1 & 11 & $\begin{array}{l}176 \pm \\
72\end{array}$ & 158 & $\begin{array}{l}108- \\
337\end{array}$ & $0(0.0 \%)$ & $2(18.2 \%)$ & $9(81.8 \%)$ & 7 (63.6\%) & $3(27.3 \%)$ & 15.31 \\
\hline POD2 & 4 & $\begin{array}{l}148 \pm \\
54\end{array}$ & 163 & 70-194 & $1(25.0 \%)$ & $0(0.0 \%)$ & $3(75.0 \%)$ & $3(75.0 \%)$ & $1(25.0 \%)$ & \\
\hline POD3 & 6 & $\begin{array}{l}111 \pm \\
12\end{array}$ & 113 & $90-122$ & 1 (16.7\%) & $5(83.3 \%)$ & $0(0.0 \%)$ & $0(0.0 \%)$ & $0(0.0 \%)$ & \\
\hline POD4 & 4 & $\begin{array}{l}148 \pm \\
39\end{array}$ & 160 & $92-180$ & $1(25.0 \%)$ & $0(0.0 \%)$ & $3(75.0 \%)$ & $3(75.0 \%)$ & $1(25.0 \%)$ & \\
\hline POD5 & 4 & $\begin{array}{l}168 \pm \\
65\end{array}$ & 171 & $\begin{array}{l}101- \\
230\end{array}$ & $0(0.0 \%)$ & $2(50.0 \%)$ & $2(50.0 \%)$ & $2(50.0 \%)$ & $2(50.0 \%)$ & \\
\hline POD6 & 4 & $\begin{array}{l}163 \pm \\
32\end{array}$ & 171 & $\begin{array}{l}122- \\
189\end{array}$ & $0(0.0 \%)$ & $1(25.0 \%)$ & $3(75.0 \%)$ & $3(75.0 \%)$ & $2(50.0 \%)$ & \\
\hline \multicolumn{11}{|c|}{ Non-diabetic } \\
\hline PRD & 52 & $88 \pm 12$ & 86 & $65-121$ & 44 (84.6\%) & 8 (15.4\%) & $0(0.0 \%)$ & $0(0.0 \%)$ & $0(0.0 \%)$ & \\
\hline POD1 & 42 & $\begin{array}{l}121 \pm \\
36\end{array}$ & 111 & $76-281$ & 13 (31.0\%) & $12(28.6 \%)$ & 17 (40.5\%) & $9(21.4 \%)$ & $3(7.1 \%)$ & 12.83 \\
\hline POD2 & 13 & $99 \pm 27$ & 101 & $68-176$ & $6(46.2 \%)$ & $6(46.2 \%)$ & $1(7.7 \%)$ & $1(7.7 \%)$ & $0(0.0 \%)$ & \\
\hline POD3 & 10 & $\begin{array}{l}104 \pm \\
32\end{array}$ & 88 & $77-184$ & 7 (70.0\%) & $1(10.0 \%)$ & $2(20.0 \%)$ & $1(10.0 \%)$ & $1(10.0 \%)$ & \\
\hline POD4 & 11 & $93 \pm 15$ & 90 & $74-119$ & 8 (72.7\%) & $3(27.3 \%)$ & $0(0.0 \%)$ & $0(0.0 \%)$ & $0(0.0 \%)$ & \\
\hline POD5 & 7 & $87 \pm 15$ & 85 & $65-112$ & $6(85.7 \%)$ & $1(14.3 \%)$ & $0(0.0 \%)$ & $0(0.0 \%)$ & $0(0.0 \%)$ & \\
\hline POD6 & 4 & $88 \pm 8$ & 89 & $77-95$ & $4(100.0 \%)$ & $0(0.0 \%)$ & $0(0.0 \%)$ & $0(0.0 \%)$ & $0(0.0 \%)$ & \\
\hline
\end{tabular}

$P R D$ pre-operative day, $P O D$ post-operative day, $S D$ standard deviation

${ }^{a}$ Data are presented as the number (percentage) of measurement

bost-operative glycemic variability was assessed using a coefficient of variation (the ratio of the standard deviation to the mean glucose level)

hyperglycemia in both diabetic and non-diabetic patients [14]. However, the above studies lacked multiple consecutive monitoring of postoperative FBG, and there is no consensus regarding how FBG varies perioperatively. While their data were limited to $24 \mathrm{~h}$ after surgery, our study included not only the observation of the glucose fluctuation in PRDs but also for 6 days postoperatively. In our study, the peak FBG appeared in the first $24 \mathrm{~h}$ 




B

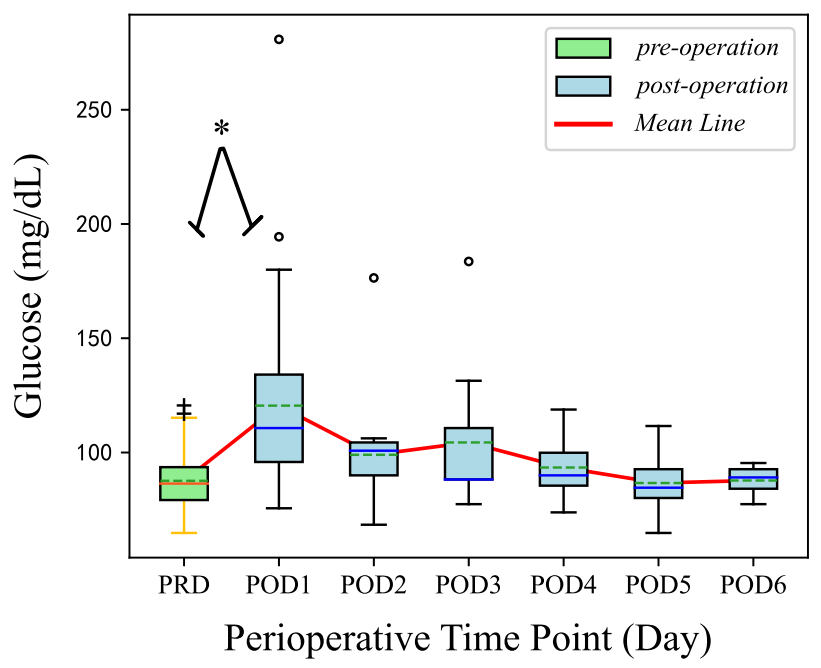

C

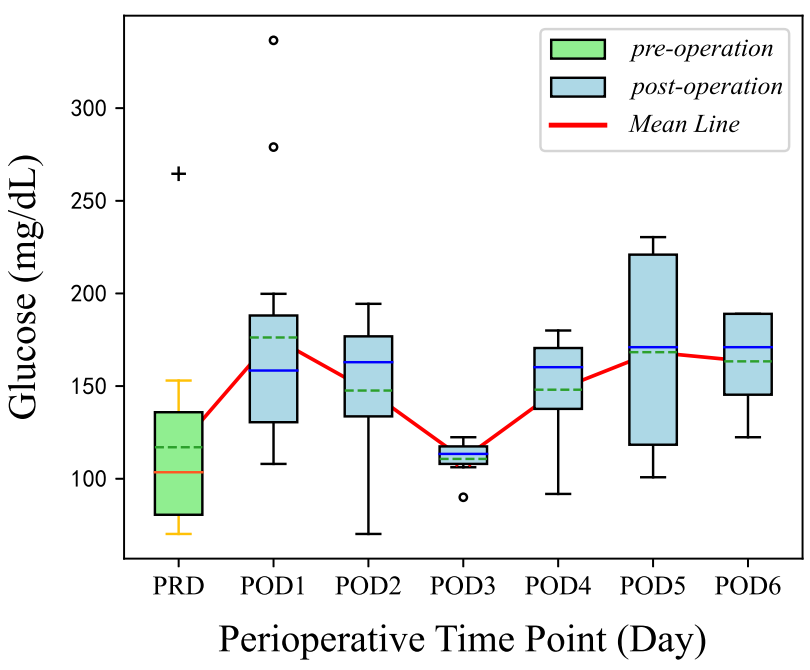

Fig. 2 a-c Boxplots of FBG for each time point across patients with revision surgery. a The fluctuation of FBG for each time point in patients with revision surgery. b, c The fluctuation of FBG for each time point in patients with revision surgery grouped by diabetic status. The asterisks indicate a significant difference between groups. The $P$ values were calculated using the Kruskal-Wallis $H$ test and corrected by Bonferroni analysis

after surgery in the primary and revision groups. Our findings suggest that the FBG for TJA patients remains within normal limits before surgery and increases substantially within the first day of TJA, and then postoperative FBG decreases mildly and tends toward normal in the non-diabetic patients within the primary group. Various factors could contribute to the elevation of blood glucose levels postoperatively, including medications, physiologic stress response, and hormones $[4,30]$. More importantly, the episodes of hyperglycemia would provide an optimal medium for bacterial growth, alter the immune response, and subsequently impair the capability of the host to battle infection, making patients more susceptible to infection postoperatively [31,
32]. Regardless of the etiology and pathophysiology of postoperative hyperglycemia, several studies have demonstrated the association between hyperglycemia in POD1 and postoperative complications $[4,19,21]$, which was in line with our findings. Kheir et al. revealed that blood glucose levels in POD1 were significantly associated with PJI, and the risk of PJI increased linearly when blood glucose levels > $115 \mathrm{mg} / \mathrm{dL}$. Furthermore, they set a glucose level of 137 $\mathrm{mg} / \mathrm{dL}$ as the optimal threshold to reduce the likelihood of PJI [4]. Another study demonstrated that significantly higher blood glucose levels in POD1 were observed in infected patients. Interestingly, non-diabetic patients with blood glucose values of $>140 \mathrm{mg} / \mathrm{dL}$ in POD1 were more 
than 3 times likely to develop PJI than those with equivalent glucose levels in the DM group [19]. However, further studies are needed to monitor the blood glucose levels after TJA in a continuous manner, aiming to determine the most sensitive time point in detecting hyperglycemia.

On the other hand, previous studies have suggested that clinicians should gain better control of glucose levels below the cutoff values to minimize the risk of postoperative complications [4, 13, 16, 19, 21]. CDC guidelines, published in 2017, stipulate that it is better to maintain blood glucose levels at less than $200 \mathrm{mg} / \mathrm{dL}$ for all patients during surgery, regardless of diabetic status [16]. Kwon et al. studied the relationship between perioperative hyperglycemia and outcomes in general surgery. They revealed that glucose levels maintained below $130 \mathrm{mg} / \mathrm{dL}$ had promising outcomes [21]. Kheir et al. found that hyperglycemia was associated with PJI, with an optimal cutoff of $137 \mathrm{mg} / \mathrm{dL}$ [4]. Mraovic et al. investigated the association between hyperglycemia and infection after TJA and found that postoperative blood glucose values of $>140 \mathrm{mg} / \mathrm{dL}$ doubled the risk of infection in patients with TJA [19]. Kremers et al. demonstrated a significantly higher risk of PJI among patients with perioperative hyperglycemia (blood glucose value $>180 \mathrm{mg} / \mathrm{dL}$ ) [13]. In our study, we classified hyperglycemia into different categories. For both the primary and revision groups, the FBG increased significantly at POD1, and the total number of hyperglycemic patients was the highest at POD1 among all measured days, indicating that POD1 was the most critical and sensitive day for clinicians to monitor FBG. At POD1, FBG in many patients was beyond the cutoff values, which suggested an increased risk of postoperative complications based on previous evidence $[4,13,16,19,21]$. Notably, controlling and maintaining glucose levels under thresholds was likely to reduce complications, which was supported by previous studies [21, 33]. Gallagher et al. pointed out that subcutaneous insulin intervention was both effective and safe for the management of postoperative hyperglycemia in TJA patients with or without DM, and the rate of developing PJI was remarkedly low by controlling hyperglycemia in a timely manner [33]. Besides investigations in the arthroplasty literature, a study regarding the general surgery as well verified the evidence that implementing insulin to lower blood glucose levels in hyperglycemic patients postoperatively ameliorates the risk of postoperative complications [21]. Therefore, clinicians should identify hyperglycemic patients by earlier monitoring after TJA and be aware that well controlling of blood glucose at POD1 can minimize the duration that the patient spends in a hyperglycemic state, which could decrease postoperative complications and improve clinical outcomes.

Understanding blood glucose variability could help doctors monitor and maintain blood glucose at stable levels [6]. Maeda et al. performed continuous glucose monitoring analysis in 20 patients who underwent THA or TKA and found that higher blood glucose levels and larger fluctuations were detected postoperatively, especially until POD2 [34]. Shohat et al. focused on the associations between hyperglycemia and adverse outcomes in patients who underwent orthopedic surgery and TJA, and they demonstrated that higher glucose variability postoperatively was associated with increased rate of complications [6, 25]. However, the extent of FBG fluctuation remains unknown. In our study, from PODs 1 to 6, the FBG gradually decreased and became stable in non-diabetic patients in the primary group. For diabetics in the primary group and diabetics and nondiabetics in the revision group, the FBG varied dramatically in the postoperative period. Greater fluctuation of FBG was observed in patients with DM and those in the revision group. Hence, the FBG of patients with DM and those undergoing revision surgery should be carefully monitored to ensure prompt detection and control of hyperglycemia.

To our knowledge, our study is the first to continuously monitor and examine the fluctuation of FBG over almost a week after TJA. However, there are some limitations to this study. First, not all confounding variables that could impact blood glucose, such as the body mass index, were considered. Second, this is a retrospective study, and not all the patients had FBG measured at each time point. Postprandial glucose and HbA1c could not be studied due to the lack of relevant extractable medical information. Third, compared to the primary group, the number of patients in the revision group was relatively small. Future studies assessing a larger cohort of revision patients and measuring glucose values at fixed intervals would be valuable to gain further insight and better patient representation.

\section{Conclusions}

Our study highlights that POD1 had the highest FBG levels and proportion of patients with hyperglycemia perioperatively. Greater attention should be paid to frequent measurement of FBG in patients with TJA after surgery, especially for patients who have DM or those who undergo revision surgery because the fluctuation of FBG was higher among such patients in our study. Clinicians should be aware of the incidence of perioperative hyperglycemia during fasting, and strategies to regulate glucose levels must be developed and implemented to avoid postoperative complications. Prospective multicenter studies are needed to further elucidate the value of detecting and controlling postoperative hyperglycemia, and thus diminish postoperative complications in patients with TJA.

\section{Abbreviations}

ADA: American Diabetes Association; CDC: Centers for Disease Control; DM: Diabetes mellitus; PJI: Periprosthetic joint infection; SSI: Surgical site infection; THA: Total hip arthroplasty; TKA: Total knee arthroplasty; TJA: Total joint arthroplasty; POD: Postoperative day; PRD: Preoperative day;

CV: Coefficient of variation 


\section{Acknowledgements}

We gratefully acknowledge the support of all doctors in our department.

\section{Authors' contributions}

YY, ZZ, and PS contributed to the idea and design of the study. YY, BP, MG, GX, WC, and LZ contributed to data collection, data analysis, and drafted the manuscript. All authors contributed to the interpretation of results and manuscript revision. All authors read and approved the final manuscript.

\section{Funding}

This work was supported by the Natural Science Foundation of Guangdong Province [grant number: 2017A030313593].

\section{Availability of data and materials}

The datasets used during the current study are available from the corresponding author on reasonable request.

\section{Ethics approval and consent to participate}

The study was approved by the Institutional Review Board of First Affiliated Hospital of Sun Yat-sen University. The requirement for informed consent was waived due to the retrospective design of our study.

\section{Consent for publication}

Not applicable.

\section{Competing interests}

There are no conflicts of interest to declare.

Received: 1 June 2020 Accepted: 20 October 2020

Published online: 05 November 2020

\section{References}

1. Parvizi J, Tan TL, Goswami K, Higuera C, Della Valle C, Chen AF, et al. The 2018 definition of periprosthetic hip and knee infection: an evidence-based and validated criteria. J Arthroplasty. 2018;33:1309-14.e2.

2. Amanatullah D, Dennis D, Oltra EG, Marcelino Gomes LS, Goodman SB, Hamlin B, et al. Hip and knee section, diagnosis, definitions: proceedings of international consensus on orthopedic infections. J Arthroplasty. 2019;34:S329-S37.

3. Huotari $\mathrm{K}$, Peltola $\mathrm{M}$, Jamsen $\mathrm{E}$. The incidence of late prosthetic joint infections: a registry-based study of 112,708 primary hip and knee replacements. Acta Orthop. 2015;86:321-5.

4. Kheir MM, Tan TL, Kheir M, Maltenfort MG, Chen AF. Postoperative blood glucose levels predict infection after total joint arthroplasty. J Bone Joint Surg Am. 2018;100:1423-31.

5. Eka A, Chen AF. Patient-related medical risk factors for periprosthetic joint infection of the hip and knee. Ann Transl Med. 2015;3:233.

6. Shohat N, Restrepo C, Allierezaie A, Tarabichi M, Goel R, Parvizi J. Increased postoperative glucose variability is associated with adverse outcomes following total joint arthroplasty. J Bone Joint Surg Am. 2018;100:1110-7.

7. Mediouni M, Madiouni R, Gardner M, Vaughan N. Translational medicine: challenges and new orthopaedic vision (Mediouni-model). Curr Orthop Pract. 2020:31:196-200.

8. Mediouni M, Schlatterer DR, Madry H, Cucchiarini M, Rai B. A review of translational medicine. The future paradigm: how can we connect the orthopedic dots better? Curr Med Res Opin. 2018;34:1217-29.

9. Mediouni M. A new generation of orthopaedic surgeons: "T-model". Curr Orthop Pract. 2019:30:444-5.

10. Maoz G, Phillips M, Bosco J, Slover J, Stachel A, Inneh I, et al. The Otto Aufranc award: modifiable versus nonmodifiable risk factors for infection after hip arthroplasty. Clin Orthop Relat Res. 2015;473:453-9.

11. Ferrera HK, Jones TE, Schudrowitz NJ, Collins JE, Lichstein PM, Shaner JL, et al. Perioperative dietary restriction of carbohydrates in the management of blood glucose levels in patients undergoing total knee replacement. J Arthroplasty. 2019:34:1 105-9.

12. He Y, Xiao J, Shi Z, He J, Li T. Supplementation of enteral nutritional powder decreases surgical site infection, prosthetic joint infection, and readmission after hip arthroplasty in geriatric femoral neck fracture with hypoalbuminemia. J Orthop Surg Res. 2019;14:292.

13. Kremers HM, Lewallen LW, Mabry TM, Berry DJ, Berbari EF, Osmon DR. Diabetes mellitus, hyperglycemia, hemoglobin A1c and the risk of prosthetic joint infections in total hip and knee arthroplasty. J Arthroplasty. 2015;30:439-43.
14. Varady NH, Schwab PE, Jones T, Collins JE, Fitz W, Chen AF. Optimal timing of glucose measurements after total joint arthroplasty. J Arthroplasty. 2019; 34:S152-S8.

15. Cui G, Zhang T, Ren F, Feng WM, Yao Y, Cui J, et al. High blood glucose levels correlate with tumor malignancy in colorectal cancer patients. Med Sci Monit. 2015;21:3825-33.

16. Berríos-Torres SI, Umscheid CA, Bratzler DW, Leas B, Stone EC, Kelz RR, et al. Centers for disease control and prevention guideline for the prevention of surgical site infection, 2017. JAMA surgery. 2017;152:784-91.

17. Parvizi J, Zmistowski B, Berbari EF, Bauer TW, Springer BD, Della Valle CJ, et al. New definition for periprosthetic joint infection: from the workgroup of the musculoskeletal infection society. Clin Orthop Relat Res. 2011;469:2992.

18. Chamberlain JJ, Johnson EL, Leal S, Rhinehart AS, Shubrook JH, Peterson L. Cardiovascular disease and risk management: review of the American Diabetes Association Standards of Medical Care in diabetes 2018. Ann Intern Med. 2018;168:640-50.

19. Mraovic B, Suh D, Jacovides C, Parvizi J. Perioperative hyperglycemia and postoperative infection after lower limb arthroplasty. J Diabetes Sci Technol. 2011;5:412-8.

20. Chrastil J, Anderson MB, Stevens V, Anand R, Peters CL, Pelt CE. Is hemoglobin A1c or perioperative hyperglycemia predictive of periprosthetic joint infection or death following primary total joint arthroplasty? J Arthroplasty. 2015;30:1197-202.

21. Kwon S, Thompson R, Dellinger P, Yanez D, Farrohki E, Flum D. Importance of perioperative glycemic control in general surgery: a report from the surgical care and outcomes assessment program. Ann Surg. 2013;257:8-14.

22. King JT, Goulet JL, Perkal MF, Rosenthal RA. Glycemic control and infections in patients with diabetes undergoing noncardiac surgery. Ann Surg. 2011; 253:158-65.

23. Siegelaar SE, Holleman F, Hoekstra JB, DeVries JH. Glucose variability; does it matter? Endocr Rev. 2010;31:171-82.

24. Rodbard D. Clinical interpretation of indices of quality of glycemic control and glycemic variability. Postgrad Med. 2011;123:107-18.

25. Shohat N, Foltz C, Restrepo C, Goswami K, Tan T, Parvizi J. Increased postoperative glucose variability is associated with adverse outcomes following orthopaedic surgery. Bone Joint J. 2018;100-B:1125-32.

26. Shohat N, Muhsen K, Gilat R, Rondon AJ, Chen AF, Parvizi J. Inadequate glycemic control is associated with increased surgical site infection in total joint arthroplasty: a systematic review and meta-analysis. J Arthroplasty. 2018;33:2312-21.e3.

27. Kremers HM, Schleck CD, Lewallen EA, Larson DR, Van Wijnen AJ, Lewallen DG. Diabetes mellitus and hyperglycemia and the risk of aseptic loosening in total joint arthroplasty. J Arthroplasty. 2017;32:S251-s3.

28. Pomposelli JJ, Baxter JK, Babineau TJ, Pomfret EA, Driscoll DF, Forse RA, et al. Early postoperative glucose control predicts nosocomial infection rate in diabetic patients. JPEN J Parenter Enteral Nutr. 1998;22:77-81.

29. Furnary AP, Zerr KJ, Grunkemeier GL, Starr A. Continuous intravenous insulin infusion reduces the incidence of deep sternal wound infection in diabetic patients after cardiac surgical procedures. Ann Thorac Surg. 1999;67:352-60 discussion 60-2.

30. Perez A, Jansen-Chaparro S, Saigi I, Bernal-Lopez MR, Minambres I, GomezHuelgas R. Glucocorticoid-induced hyperglycemia. J Diabetes. 2014;6:9-20.

31. Turina M, Fry DE, Polk HC Jr. Acute hyperglycemia and the innate immune system: clinical, cellular, and molecular aspects. Crit Care Med. 2005;33:1624-33.

32. Xiu F, Stanojcic M, Diao L, Jeschke MG. Stress hyperglycemia, insulin treatment, and innate immune cells. Int J Endocrinol. 2014;2014:486403.

33. Gallagher JM, Erich RA, Gattermeyer R, Beam KK. Postoperative hyperglycemia can be safely and effectively controlled in both diabetic and nondiabetic patients with use of a subcutaneous insulin protocol. JBJS Open Access. 2017;2:e0008.

34. Maeda Y, Nakamura N, Tsujimoto T, Sugano N. Higher blood glucose and larger fluctuations detected postoperatively using continuous glucose monitoring: a preliminary study following total knee or hip arthroplasty. J Exp Orthop. 2019;6:15.

\section{Publisher's Note}

Springer Nature remains neutral with regard to jurisdictional claims in published maps and institutional affiliations. 